quatrième série-tome $44 \quad$ fascicule $4 \quad$ juillet-août 2011

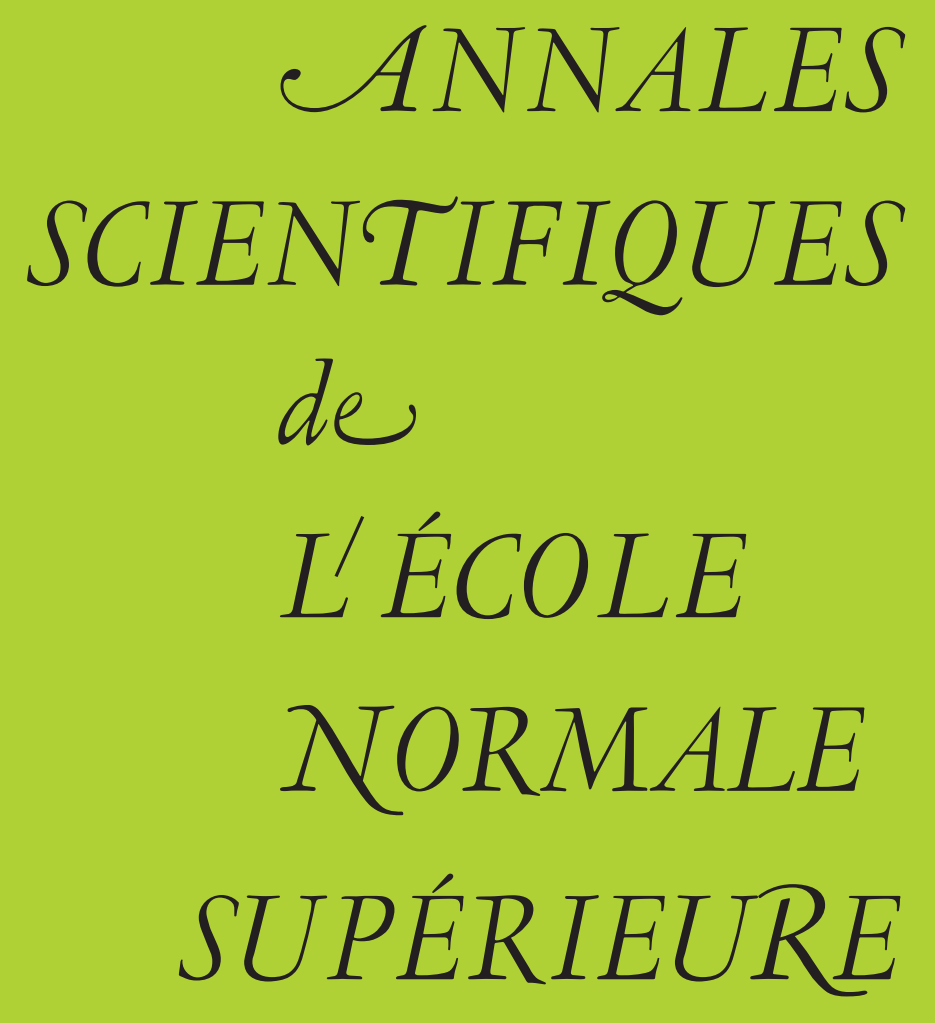

Amnon NEEMAN

Explicit cogenerators for the bomotopy category

of projective modules over a ring

SOCIÉTÉ MATHÉMATIQUE DE FRANCE 
Ann. Scient. Éc. Norm. Sup.

$4^{\text {e }}$ série, t. 44, 2011, p. 607 à 629

\title{
EXPLICIT COGENERATORS FOR THE HOMOTOPY CATEGORY OF PROJECTIVE MODULES OVER A RING
}

\author{
BY AMNON NEEMAN
}

Abstract. - Let $R$ be a ring. In two previous articles $[12,14]$ we studied the homotopy category $\mathbf{K}(R$-Proj) of projective $R$-modules. We produced a set of generators for this category, proved that the category is $\aleph_{1}$-compactly generated for any ring $R$, and showed that it need not always be compactly generated, but is for sufficiently nice $R$. We furthermore analyzed the inclusion $j_{!}: \mathbf{K}(R$-Proj $) \longrightarrow \mathbf{K}(R$-Flat $)$ and the orthogonal subcategory $\&=\mathbf{K}(R \text {-Proj })^{\perp}$. And we even showed that the inclusion $\& \longrightarrow \mathbf{K}(R$-Flat $)$ has a right adjoint; this forces some natural map to be an equivalence $\mathbf{K}(R$-Proj $) \longrightarrow \phi^{\perp}$.

In this article we produce a set of cogenerators for $\mathbf{K}(R$-Proj $)$. More accurately, this set of cogenerators naturally lies in the equivalent $\phi^{\perp} \cong \mathbf{K}(R$-Proj $)$; it can be used to give yet another proof of the fact that the inclusion $\& \longrightarrow \mathbf{K}(R$-Flat $)$ has a right adjoint. But by now several proofs of this fact already exist.

RÉsumé. - Soit $R$ un anneau. Dans deux articles antérieurs [12, 14], on a étudié la catégorie d'homotopie $\mathbf{K}(R$-Proj) des $R$-modules projectifs. On a construit un ensemble de générateurs pour cette catégorie et on a démontré que la catégorie est compactement générée de niveau $\aleph_{1}$ pour chaque anneau $R$, mais qu'elle n'est pas toujours compactement générée. Toutefois, pour $R$ un anneau suffisamment raisonnable, la catégorie $\mathbf{K}(R$-Proj) est compactement générée. On a étudié l'inclusion $j_{!}: \mathbf{K}(R$-Proj $) \longrightarrow \mathbf{K}(R$-Flat $)$ et la sous-catégorie orthogonale $\&=\mathbf{K}(R \text {-Proj })^{\perp}$. On a même montré que l'inclusion $\& \longrightarrow \mathbf{K}(R$-Flat $)$ admet un adjoint à droite ; il s'ensuit qu'une certaine application naturelle $\mathbf{K}(R$-Proj $) \longrightarrow \phi^{\perp}$ est une équivalence.

Dans le présent article, on produit un ensemble de cogénérateurs pour $\mathbf{K}(R$-Proj). Plus précisément, cet ensemble de cogénérateurs appartient naturellement à la catégorie équivalente $\delta^{\perp} \cong \mathbf{K}(R$-Proj); on peut l'utiliser pour obtenir une nouvelle démonstration du fait que l'inclusion $\& \longrightarrow \mathbf{K}(R$-Flat $)$ admet un adjoint à droite. Mais il y a déjà plusieurs autres démonstrations de ce fait.

The research was partly supported by the Australian Research Council. 


\section{Introduction}

Let $\mathscr{T}$ be a triangulated category with products. A subcategory $\mathscr{S} \subset \mathscr{T}$ is called colocalizing if it is triangulated and closed under products. Given any class of objects $T \subset \mathscr{T}$, the smallest colocalizing subcategory containing $T$ will be denoted $\operatorname{Coloc}(T)$, and referred to as the colocalizing subcategory cogenerated by $T$. If $\operatorname{Coloc}(T)=\mathscr{T}$, then we say that $T$ cogenerates $\mathscr{T}$. If $\mathscr{T}$ has coproducts (that is, $\mathscr{T}^{\text {op }}$ has products) then we can dualize: a class of objects $T \subset \mathscr{T}$ is said to generate $\mathscr{T}$ if it cogenerates $\mathscr{T}^{\text {op }}$.

For various reasons it is interesting to try to find sets of objects (as opposed to classes) $T \subset \mathscr{T}$ which cogenerate $\mathscr{T}$. One reason is that all known proofs of the Brown representability theorem depend on producing sets of generators or, dually, of cogenerators. Embarrassingly, the situation is not symmetric. We often know how to produce a set of generators for some category $\mathscr{T}$, without having the foggiest clue whether the category has a set of cogenerators. Thus Brown's original proof of his representability theorem in [2], which looked at the case where $\mathscr{T}$ was the homotopy category of spectra, depended on the fact that the spheres are compact generators. It took 36 years before anyone noticed that this category has a sufficiently nice set of cogenerators so that Brown representability also holds for the dual; see [10].

By now it is known how to produce a set of cogenerators in any compactly generated triangulated category; there are two somewhat different treatments of the same set of cogenerators in Krause [6] and in [11], together with proofs that its existence implies Brown representability for the dual. Assuming the category $\mathscr{T}$ has a Rosický functor then there is a completely different construction of a set of cogenerators in [13], and, once again, an argument showing that these cogenerators are nice enough to force Brown representability to hold in the dual. But it is not clear how useful this observation is; ever since Rosický retracted his result of [15], we know of relatively few examples where we can directly produce Rosický functors. Unfortunately this is all we know at present, there is no known general method to produce cogenerators in categories that happen not to be compactly generated. Hence the interest of this article: we give a construction of cogenerators in categories known to be well generated but not compactly generated.

Let $R$ be an associative ring with a unit. Let $\mathbf{K}(R-$ Proj) be the homotopy category of chain complexes of projective $R$-modules. The main result of this article amounts to producing an explicit set of cogenerators. But there is a technical wrinkle that we should now explain: the actual cogenerators we choose naturally lie not in $\mathbf{K}(R-$ Proj) but in an equivalent category that we will often denote by $\mathscr{S}^{\perp}$. It is now time to elaborate.

In [12, Proposition 8.1] we proved that the natural inclusion $j_{!}: \mathbf{K}(R-$ Proj $) \longrightarrow \mathbf{K}(R-$ Flat $)$ has a right adjoint $j^{*}: \mathbf{K}(R-$ Flat $) \longrightarrow \mathbf{K}(R$-Proj). In [14, Theorem 3.1] we showed that the functor $j^{*}$ has a right adjoint $j_{*}: \mathbf{K}(R-$ Proj $) \longrightarrow \mathbf{K}(R$-Flat $)$. The functor $j_{\text {! }}$ is obviously fully faithful and it follows formally, from general nonsense about triangulated categories, that so is the functor $j_{*}$. Our cogenerators can naturally be described as objects in the essential image of $j_{*}$.

Let us rephrase this a little. We have a fully faithful functor $j_{!}: \mathbf{K}(R-$ Proj $) \longrightarrow \mathbf{K}(R-$ Flat $)$, namely the obvious embedding, and it possesses a right adjoint $j^{*}$. Define $\mathscr{S}=\mathbf{K}(R-\text { Proj })^{\perp}$ to be the full subcategory of $\mathbf{K}(R-$ Flat $)$ whose objects are

$$
\mathrm{Ob}(\mathscr{S})=\left\{y \in \mathbf{K}(R-\text { Flat }) \mid \operatorname{Hom}\left(j_{!} x, y\right)=0 \quad \forall x \in \mathbf{K}(R-\text { Proj })\right\} .
$$

4 e SÉRIE - TOME $44-2011-$ No $^{\circ}$ 
Then the essential image of the functor $j_{*}: \mathbf{K}(R-$ Proj $) \longrightarrow \mathbf{K}(R$-Flat $)$ is precisely the subcategory $\mathscr{S}^{\perp} \subset \mathbf{K}(R$-Flat). Our main theorem proves that a certain explicit set of objects in $\mathscr{S}^{\perp}$ cogenerates. In order to write down these explicit cogenerators we remind the reader:

REMINDER 0.1. - The natural inclusion $\mathbf{K}(R$-Flat $) \longrightarrow \mathbf{K}(R$-Mod $)$ has a right adjoint $J: \mathbf{K}(R-$ Mod $) \longrightarrow \mathbf{K}(R-$ Flat $)$; the first proof appeared in [14, Theorem 3.2], but since then there have been other proofs of more general results: see Bravo, Enochs, Iacob, Jenda and Rada [1, Theorem 3.5 and the third Example in §4], Krause [8, Corollary 3.4], and Saorín and Štovíček [16, Proposition 4.12].

The following statement summarizes the main results of the paper:

Theorem 0.2. - Let the notation be as above. In particular, let $\mathscr{S}=\mathbf{K}(R-\text { Proj })^{\perp}$ be the category of all $\mathbf{K}(R-$ Proj)-local objects in $\mathbf{K}(R$-Flat $)$. Then the objects of $\mathscr{S}^{\perp}=\left\{\mathbf{K}(R \text {-Proj })^{\perp}\right\}^{\perp}$ are cogenerated by the chain complexes $J\left(\mathscr{H}\right.$ om $\left._{\mathbb{Z}}(I, \mathbb{Q} / \mathbb{Z})\right) \in$ $\mathbf{K}(R-$ Flat $)$, where

1. The functor $J$ is the right adjoint to the inclusion $\mathbf{K}(R-$ Flat $) \longrightarrow \mathbf{K}(R-\mathrm{Mod})$.

2. I runs over the bounded below chain complexes of injective right $R$-modules, which satisfy the following two conditions:

(a) All but finitely many of the groups $H^{i}(I)$ vanish.

(b) For all $i, H^{i}(I)$ is isomorphic to a subquotient of a finitely generated, projective right $R$-module.

The proof may be found in Theorem 4.7.

REMARK 0.3. - In the process of proving Theorem 4.7 we discover that we also give yet another proof of the existence of a right adjoint to the natural inclusion $i_{*}: \mathscr{S} \longrightarrow \mathbf{K}(R$-Flat $)$. More explicitly the argument goes as follows: it is easy to show that the objects $J\left(\mathscr{H}_{0} m_{\mathbb{Z}}(I, \mathbb{Q} / \mathbb{Z})\right)$ all lie in $\mathscr{S}^{\perp}$; see Remark 2.7. One immediately deduces the inclusion $\operatorname{Coloc}(S) \subset \mathscr{S}^{\perp}$. Given an object $y \in \mathbf{K}(R$-Flat) we will show how to construct a triangle

$$
s \longrightarrow y \longrightarrow t \longrightarrow \Sigma s
$$

with $s \in \mathscr{S}$ and $t \in \operatorname{Coloc}(S)$; this will automatically prove both the existence of the right adjoint to the functor $i_{*}$ and the fact that $\operatorname{Coloc}(S)=\mathscr{S}^{\perp}$.

REMARK 0.4. - When the ring $R$ is right coherent, we find that the proof of Theorem 4.7 can be done without making use of the functor $J$. The argument of this paper simplifies substantially when $R$ is right coherent. Part of the reason is that, when $R$ is right coherent, the category $K(R-$ Proj $)$ is compactly generated, and for compactly generated categories there is a standard way to construct cogenerators. The standard cogenerators are the BrownComenetz duals of the compact objects; in $\$ 5$ we will see that, for right coherent $R$, the set of cogenerators we give in Theorem 0.2 includes the standard ones.

The remarkable feature of our construction of cogenerators is its generality. For general $R$ the category $K(R-$ Proj $)$ is only well generated and not compactly generated, and there is no known procedure to construct cogenerators. It is known that the recipe that works for 
compactly generated categories does not generalize; this can be restated as saying that a certain abelian category does not have enough injectives, see [11, Appendix D.2]. What we produce here amounts to the first known example of cogenerators in a non-compactlygenerated but well generated triangulated category.

REMARK 0.5. - In the special case where $R$ is commutative, noetherian and of finite Krull dimension there is already a discussion of $\mathscr{S}^{\perp}$ in the literature; see Enochs and Garcia [3, Theorem 4.6]. We should also mention the growing literature on related topics: see Bravo, Enochs, Iacob, Jenda and Rada [1], Jørgensen [5], Iyengar and Krause [4], Krause [7, 8], Murfet [9], and Saorín and Štovíček [16].

\section{Acknowledgements}

I would like to thank the referee for helpful suggestions on how to improve an earlier version.

\section{Tensor-phantom maps}

This section is devoted to technical preliminaries. The highlights are Definitions 1.1 and 1.3, as well as Lemma 1.9. The reader wishing to form an overall impression of the proof might wish to read just the two definitions and the statement of the lemma, and then proceed to $\S 2$. The current section contains the hard work in the article, but the reader might prefer to first have an idea why we bother.

Definition 1.1. - A test-complex $I$ is a bounded below chain complex of injective right $R$-modules, with $H^{i}(I)=0$ for all but finitely many $i \in \mathbb{Z}$. For those $i \in \mathbb{Z}$ for which $H^{i}(I) \neq 0$, we insist that $H^{i}(I)$ must be isomorphic to a subquotient of a finitely generated, projective right $R$-module.

REMARK 1.2. - The definition is intended to ensure that, up to homotopy equivalence, there is only a set of test-complexes. Up to isomorphism, the collection of finitely generated, projective right $R$-modules forms a set. Hence so do all their subquotients. Therefore the triangulated subcategory $\mathscr{R}$, that these subquotients generate in $\mathbf{D}^{b}(R-\mathrm{Mod})$, is essentially small. The test-complexes are injective resolutions of some of the objects in $\mathscr{R}$; there is only a set of them, up to homotopy equivalence.

Definition 1.3. - Let $Y$ and $Z$ be objects in $\mathbf{K}(R$-Flat). A morphism $f: Y \longrightarrow Z$ is called tensor-phantom if, for every test-complex $I$ as in Definition 1.1, the map

$$
I \otimes_{R} Y \stackrel{1 \otimes f}{\longrightarrow} I \otimes_{R} Z
$$

vanishes in cohomology. That is, the induced maps $H^{i}\left(I \otimes_{R} Y\right) \longrightarrow H^{i}\left(I \otimes_{R} Z\right)$ all vanish.

REMARK 1.4. - The tensor-phantom maps form an ideal, in the category $\mathbf{K}(R$-Flat). We remind the reader: this means

(i) If $g, g^{\prime}: Y \longrightarrow Z$ are two tensor-phantom maps, then $g+g^{\prime}$ is also tensor-phantom.

(ii) If $f: X \longrightarrow Y, g: Y \longrightarrow Z$ and $h: Z \longrightarrow Z^{\prime}$ are maps of chain complexes, and if $g$ is tensor-phantom, then $g f: X \longrightarrow Z$ and $h g: Y \longrightarrow Z^{\prime}$ are also tensor-phantom.

4 e SÉRIE - TOME $44-2011-$ No $^{\circ}$ 
REMINDER 1.5. - We remind the reader of [12, Construction 4.3]. In there we defined a set $T$ of objects in $\mathbf{K}(R$-Flat); the elements of $T$ are the bounded-below complexes of finitely generated projectives. More precisely, $T$ contains one representative in each $\mathbf{C}^{+}(R$-proj)-isomorphism class of objects.

Lemma 1.6. - Let $f: X \longrightarrow Z$ be a tensor-phantom map. Assume $X$ is an object in the set $T$ of Reminder 1.5, while $Z$ is any chain complex of flat modules. Then, for any integer $n \in \mathbb{Z}$, the map $f$ can be factored in $\mathbf{K}(R$-Flat) as

$$
X \longrightarrow Y \longrightarrow Z,
$$

with $Y \in T$, and where $Y^{i}=0$ for all $i<n$.

Proof. - We recall [12, Lemma 8.4]: the chain complex $Z$ is the filtered direct limit of the category $T / Z$; that is, it is the filtered direct limit of the maps $\varphi: Y \longrightarrow Z$, with $Y \in T$. This means that, for any chain complex $I$ of right $R$-modules, the chain complex $I \otimes_{R} Z$ is the filtered direct limit of all the maps $1 \otimes \varphi: I \otimes_{R} Y \longrightarrow I \otimes_{R} Z$, and furthermore that $H^{i}\left(I \otimes_{R} Z\right)$ is the filtered direct limit of $H^{i}(1 \otimes \varphi): H^{i}\left(I \otimes_{R} Y\right) \longrightarrow H^{i}\left(I \otimes_{R} Z\right)$.

We are given a map $f: X \longrightarrow Z$, with $X \in T$. We know that this map is tensor-phantom. This means that, for all test-complexes $I$, the induced maps

$$
H^{i}(1 \otimes f): H^{i}\left(I \otimes_{R} X\right) \longrightarrow H^{i}\left(I \otimes_{R} Z\right)
$$

vanish for all $i \in \mathbb{Z}$. By the paragraph above we conclude that, for any element $h \in H^{i}\left(I \otimes_{R} X\right)$, there exists a factorization of $f: X \longrightarrow Z$ as $X \stackrel{\alpha}{\longrightarrow} Y \stackrel{\beta}{\longrightarrow} Z$, with $Y \in T$, and so that $h$ is killed already by the map $H^{i}(1 \otimes \alpha)$ in the composite

$$
H^{i}\left(I \otimes_{R} X\right) \stackrel{H^{i}(1 \otimes \alpha)}{\longrightarrow} H^{i}\left(I \otimes_{R} Y\right) \stackrel{H^{i}(1 \otimes \beta)}{\longrightarrow} H^{i}\left(I \otimes_{R} Z\right) .
$$

The proof will be based on making an intelligent choice of the test complex $I$, and of the element $h \in H^{i}\left(I \otimes_{R} X\right)$.

Now $X$ belongs to $T$; it is a bounded below chain complex of left $R$-modules. This makes $X^{*}=\mathscr{H}_{\mathrm{O}} \mathrm{m}_{R}(X, R)$ into a bounded above chain complex of right $R$-modules. The $t$-structure truncation $\left\{X^{*}\right\}^{>-n}$ is a bounded complex of right $R$-modules. Let $I$ be an injective resolution for it. Then $I$ is a bounded below complex of right $R$-modules. Since $X^{*}$ is bounded above, and since $H^{i}\left(X^{*}\right)=H^{i}(I)$ for all $i>-n$, we conclude that $H^{i}(I)$ vanishes outside a finite range. Furthermore, $H^{i}(I)$ is isomorphic to $H^{i}\left(\left\{X^{*}\right\}^{>-n}\right)$, and $H^{i}\left(\left\{X^{*}\right\}^{>-n}\right)$ is a subquotient of the finitely generated, projective right $R$-module $\left\{X^{-i}\right\}^{*}$. That is, $I$ is a test-complex.

We have a natural map

$$
X^{*} \longrightarrow\left\{X^{*}\right\}^{>-n} \longrightarrow I,
$$

which we may view as an element $h$ in $H^{0}$ of the complex $\mathscr{H}_{\mathrm{om}}\left(X^{*}, I\right) \cong I \otimes_{R} X$. We wish to apply the observations of the first two paragraphs of the proof, to this particular $h \in H^{0}\left(I \otimes_{R} X\right)$. 
REMARK 1.7. - The reader should note that the isomorphism $I \otimes_{R} X \cong \mathscr{H}_{\mathrm{om}}\left(X^{*}, I\right)$ uses all the boundedness hypotheses. For each pair of integers $i, j \in \mathbb{Z}$, we have an isomorphism

$$
I^{j} \otimes_{R} X^{i} \cong \operatorname{Hom}_{R}\left(\operatorname{Hom}_{R}\left(X^{i}, R\right), I^{j}\right)=\operatorname{Hom}_{R}\left(\left\{X^{i}\right\}^{*}, I^{j}\right),
$$

just because $X^{i}$ is assumed finitely generated and projective. The chain complex $I \otimes_{R} X$ is formed by taking direct sums over $i+j=n$ of the groups $I^{j} \otimes_{R} X^{i}$, while in the complex $\mathscr{H}_{R}\left(X^{*}, I\right)$ we take the product of the groups $\operatorname{Hom}_{R}\left(\left\{X^{i}\right\}^{*}, I^{j}\right)$, over $j-(-i)=n$. The reason the direct sum agrees with the direct product is because, for each $n \in \mathbb{Z}$, there are only finitely many non-zero pairs $X^{i}, I^{j}$ with $i+j=n$; this is because both complexes are bounded below.

Back to the proof of Lemma 1.6. Just before Remark 1.7, we defined a test-complex $I$ and an element $h \in H^{0}\left(I \otimes_{R} X\right)$. The first couple of paragraphs, of the proof of Lemma 1.6, tell us that the map $f: X \longrightarrow Z$ must factor as $X \stackrel{\alpha}{\longrightarrow} Y \stackrel{\beta}{\longrightarrow} Z$, with $Y$ belonging to $T$, and so that $h$ is killed already by the map $H^{0}(1 \otimes \alpha)$. The naturality of the isomorphism $\mathscr{H}_{\mathrm{om}}\left(X^{*}, I\right) \cong I \otimes_{R} X$ tells us that the image of $h$ under the map

$$
H^{0}\left(\mathscr{H}_{\mathrm{om}}\left(X^{*}, I\right)\right) \longrightarrow H^{0}\left(\mathscr{H}_{\mathrm{om}}\left(Y^{*}, I\right)\right)
$$

will vanish. In other words, the composite

$$
Y^{*} \stackrel{\alpha^{*}}{\longrightarrow} X^{*} \longrightarrow\left\{X^{*}\right\}^{>-n} \longrightarrow I
$$

must be null homotopic. In the derived category the map $\left\{X^{*}\right\}^{\geq-n} \longrightarrow I$ is an isomorphism, and the composite

$$
Y^{*} \stackrel{\alpha^{*}}{\longrightarrow} X^{*} \longrightarrow\left\{X^{*}\right\}^{>-n}
$$

vanishes. This means that, in the derived category, the map $\alpha^{*}: Y^{*} \longrightarrow X^{*}$ must factor as

$$
Y^{*} \longrightarrow\left\{X^{*}\right\}^{\leq-n} \longrightarrow X^{*} \text {. }
$$

Since $Y^{*}$ is a bounded above complex of projectives, factorizations in the derived category lift to factorizations up to homotopy; the map $\alpha^{*}$ factors, up to homotopy, through the complex $\left\{X^{*}\right\}^{\leq-n}$, which vanishes in dimensions $>-n$. This means that the map $\alpha: X \longrightarrow Y$ is homotopic to a map $\alpha^{\prime}: X \longrightarrow Y$, which vanishes in degrees $<n$.

Now we appeal to [12, Lemma 4.1], which tells us that $\alpha^{\prime}: X \longrightarrow Y$ must factor as $X \longrightarrow W \longrightarrow Y$, with $W \in T$, and $W^{i}=0$ for all $i<n$.

Lemma 1.8. - As in Lemma 1.6 let $f: X \longrightarrow Z$ be a tensor-phantom map, and assume that $X$ is an object in the set $T$, while $Z$ is free to be any chain complex of flat modules. Then the map $f$ can be factored in $\mathbf{K}(R-\mathrm{Mod})$ as

$$
X \longrightarrow \coprod_{n=0}^{\infty} C_{n} \stackrel{\coprod_{n=0}^{\infty} f_{n}}{\longrightarrow} \coprod_{n=0}^{\infty} Y_{n} \longrightarrow Z,
$$

with $C_{n}$ bounded complexes, and with $Y_{n} \in T$.

$4^{\text {e }}$ SÉRIE - TOME $44-2011-$ No $^{\circ}$ 
Proof. - We have that $X$ is a bounded below chain complex

$$
\cdots \longrightarrow X^{-2} \longrightarrow X^{-1} \longrightarrow X^{0} \longrightarrow X^{1} \longrightarrow X^{2} \longrightarrow \cdots
$$

This makes $X^{*}$ the bounded above chain complex

$\cdots \longrightarrow\left\{X^{2}\right\}^{*} \longrightarrow\left\{X^{1}\right\}^{*} \longrightarrow\left\{X^{0}\right\}^{*} \longrightarrow\left\{X^{-1}\right\}^{*} \longrightarrow\left\{X^{-2}\right\}^{*} \longrightarrow \cdots$

As in the proof of [12, Proposition 7.14], we consider the $t$-structure truncations $\left\{X^{*}\right\}^{>-i}$; these are obtained by killing the cohomology groups in degrees $\leq-i$. For each $i \geq 0$ there exists a complex

$\cdots \longrightarrow W_{i}^{-i-3} \longrightarrow W_{i}^{-i-2} \longrightarrow W_{i}^{-i-1} \longrightarrow\left\{X^{i}\right\}^{*} \longrightarrow\left\{X^{i-1}\right\}^{*} \longrightarrow \cdots$

of projective right $R$-modules, which is isomorphic in the derived category $\mathbf{D}(R$-Mod) to $\left\{X^{*}\right\}^{>-i}$. Note that we are not assuming $R$ coherent, and therefore the $W_{i}^{j}$ will not, in general, be finitely generated. Choose such a complex, and call it $W_{i}$. All the complexes $W_{i}$ are bounded above complexes of projectives, and hence maps in the derived category correspond bijectively to homotopy equivalence classes of chain maps. We can choose chain maps $W_{i+1} \longrightarrow W_{i}$, lifting the canonical map in the derived category $\left\{X^{*}\right\}^{>-i-1} \longrightarrow\left\{X^{*}\right\}^{>-i}$. That is, there is a chain map

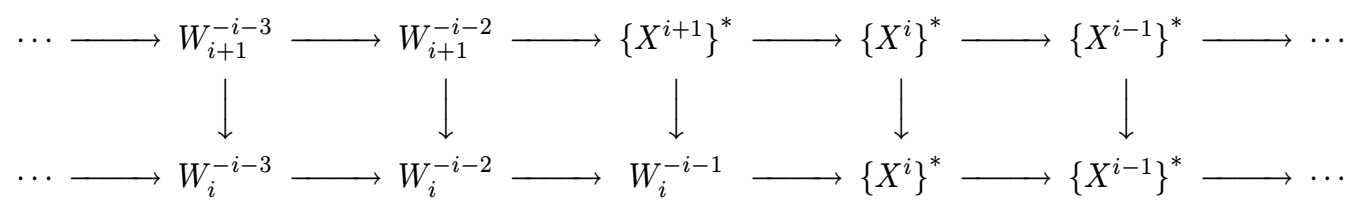

lifting the map of $t$-structure truncations. As in the proof of [12, Proposition 7.14], we leave it to the reader to check that this chain map can be chosen so that, in degrees $j \leq i$, it is just the identity map $1:\left\{X^{j}\right\}^{*} \longrightarrow\left\{X^{j}\right\}^{*}$. That is, the diagram above becomes

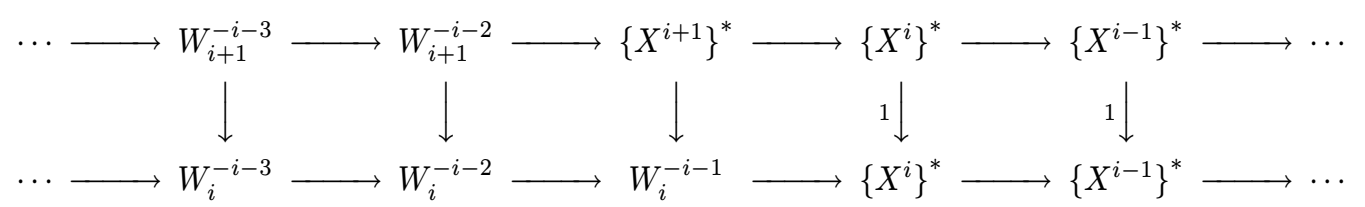

Dualizing, we have a sequence of chain maps

$$
W_{0}^{*} \longrightarrow W_{1}^{*} \longrightarrow W_{2}^{*} \longrightarrow W_{3}^{*} \longrightarrow \cdots
$$

The reader should note that these are just chain complexes of left $R$-modules. We are not claiming that the modules are projective, or even flat. But what is obvious is that the colimit of the complexes $W_{i}^{*}$ is the complex $X$; in this sequence, the $\left\{W_{i}^{*}\right\}^{j}$ stabilize to be equal to $X^{j}$ after finitely many $i$.

Next we will show that, for each $n \geq 0$, the composite $W_{n}^{*} \longrightarrow X \longrightarrow Z$ vanishes in $\mathbf{K}(R-$ Mod $)$. Lemma 1.6 tells us that the map $X \longrightarrow Z$ factors as $X \longrightarrow Y \longrightarrow Z$, with $Y \in T$, and with $Y^{i}=0$ for all $i<n$. This means that the composite

$$
Y^{*} \longrightarrow X^{*} \longrightarrow W_{n}
$$

is a map from $Y^{*}$, which vanishes in degrees $>-n$, to $W_{n}$, which is quasi-isomorphic to $\left\{X^{*}\right\}^{>-n}$. The composite must vanish in the derived category. Since $Y$ is in $T, Y^{*}$ is a bounded above complex of projectives. It follows that the composite $Y^{*} \longrightarrow W_{n}$ is null 
homotopic. Hence so is the composite $W_{n}^{*} \longrightarrow X \longrightarrow Y$, and this certainly means that the longer composite

$$
W_{n}^{*} \longrightarrow X \longrightarrow Y \longrightarrow Z
$$

must vanish in $\mathbf{K}(R-\mathrm{Mod})$.

The colimit of the sequence $W_{n}^{*}$ is $X$, and in each degree $i$ the sequence $\left\{W_{n}^{*}\right\}^{i}$ eventually stabilizes to $X^{i}$. From [12, Remark 6.3] we conclude that the chain complex $X$ is the homotopy colimit of the complexes $W_{n}^{*}$. That is, there is a distinguished triangle

$$
\coprod_{n=0}^{\infty} W_{n}^{*} \stackrel{1-s h i f t}{\longrightarrow} \coprod_{n=0}^{\infty} W_{n}^{*} \stackrel{u}{\longrightarrow} X \stackrel{v}{\longrightarrow} \coprod_{n=0}^{\infty} \Sigma W_{n}^{*}
$$

We have just shown that the composite

$$
\coprod_{n=0}^{\infty} W_{n}^{*} \stackrel{u}{\longrightarrow} X \stackrel{f}{\longrightarrow} Z
$$

vanishes, and hence the map $f: X \longrightarrow Z$ must factor as

$$
X \stackrel{v}{\longrightarrow} \coprod_{n=0}^{\infty} \Sigma W_{n}^{*} \longrightarrow Z .
$$

The next point is that we know the map $v$; we can compute it quite explicitly.

We remind the reader: the map $v$ comes about as follows. We have a short exact sequence of chain complexes

$$
0 \longrightarrow \coprod_{n=0}^{\infty} W_{n}^{*} \stackrel{1-\text { shift }}{\longrightarrow} \coprod_{n=0}^{\infty} W_{n}^{*} \stackrel{u}{\longrightarrow} X \longrightarrow 0
$$

and, degree by degree, the sequence is split. For each $i$ we are free to choose a splitting, that is a map $s^{i}: X^{i} \longrightarrow \oplus_{n=0}^{\infty}\left\{W_{n}^{*}\right\}^{i}$ splitting $u^{i}: \oplus_{n=0}^{\infty}\left\{W_{n}^{*}\right\}^{i} \longrightarrow X^{i}$. We are spoilt for choice; for all $n \geq i$, we have that $\left\{W_{n}^{*}\right\}^{i} \longrightarrow X^{i}$ is the identity. The splitting $s$ we choose is:

$$
\begin{array}{ll}
X^{i} \stackrel{1}{\longrightarrow}\left\{W_{i+2}^{*}\right\}^{i} \stackrel{\text { inclusion }}{\longrightarrow} \bigoplus_{n=0}^{\infty}\left\{W_{n}^{*}\right\}^{i} & \text { If } n>-2 \\
X^{i} \stackrel{1}{\longrightarrow}\left\{W_{0}^{*}\right\}^{i} \stackrel{\text { inclusion }}{\longrightarrow} \bigoplus_{n=0}^{\infty}\left\{W_{n}^{*}\right\}^{i} & \text { If } n \leq-2 .
\end{array}
$$

Now $s \partial-\partial s$ gives a chain map, as in the diagram below

$$
0 \longrightarrow \coprod_{n=0}^{\infty} \Sigma W_{n}^{*} \underset{1-\text { shift }}{\longrightarrow} \coprod_{n=0}^{\infty} \Sigma W_{n}^{*} \underset{u}{\longrightarrow} \Sigma X \longrightarrow 0
$$


and the composite $u \circ\{s \partial-\partial s\}$ vanishes. Hence there is a unique factorization of $s \partial-\partial s$ as below

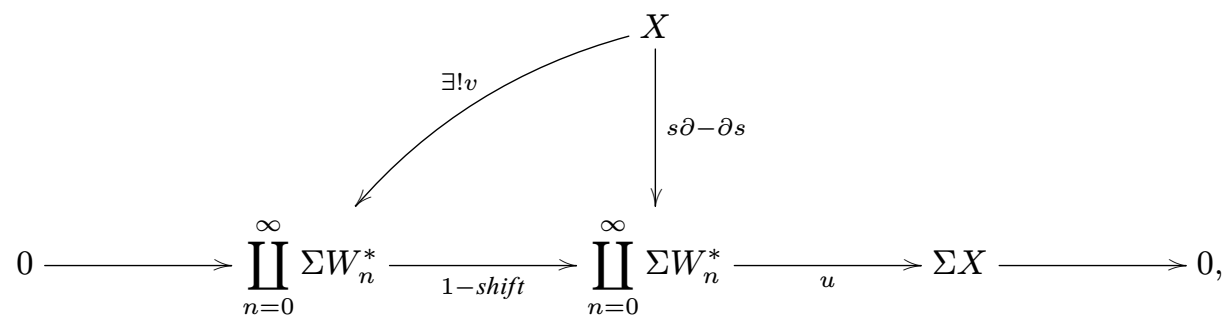

and this defines the map $v$. Changing the choice of splitting changes $v$ by a homotopy.

In our case we have chosen a splitting, and it becomes an exercise to compute $v$. The map $v$ is a map from the chain complex $X$ to a direct sum of chain complexes $\Sigma W_{n}^{*}$. I leave to the reader to check the computation; the map $X \longrightarrow \Sigma W_{n}^{*}$ comes down to

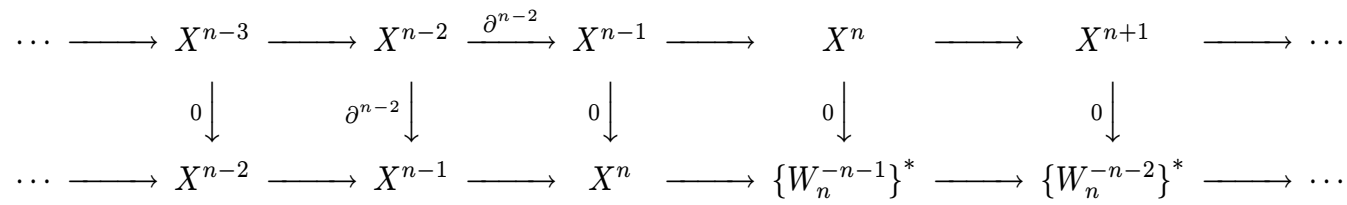

This chain map clearly factors as

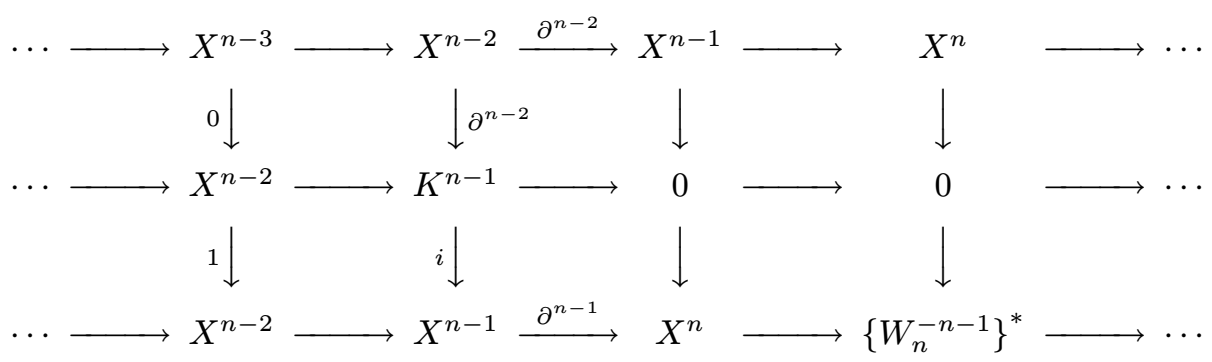

where $K^{n-1}$ is the kernel of $\partial^{n-1}: X^{n-1} \longrightarrow X^{n}$. Let $C_{n}$ be the complex

$\cdots \longrightarrow X^{n-3} \longrightarrow X^{n-2} \longrightarrow K^{n-1} \longrightarrow 0 \longrightarrow 0$

Then $C_{n}$ is a bounded complex of $R$-modules, and we have shown that the map $v$ factors as

$$
X \longrightarrow \coprod_{n=0}^{\infty} C_{n} \longrightarrow \coprod_{n=0}^{\infty} \Sigma W_{n}^{*}
$$

Now we remind the reader that, at the beginning of the proof, we showed that $f: X \longrightarrow Z$ factors as

$$
X \stackrel{v}{\longrightarrow} \coprod_{n=0}^{\infty} \Sigma W_{n}^{*} \longrightarrow Z \text {. }
$$

What we now know, about the map $v$, allows us to factor this further as

$$
X \longrightarrow \coprod_{n=0}^{\infty} C_{n} \longrightarrow \coprod_{n=0}^{\infty} \Sigma W_{n}^{*} \longrightarrow Z \text {. }
$$


This is almost what we need to prove; the lemma asserts the existence of a factorization

$$
X \longrightarrow \coprod_{n=0}^{\infty} C_{n} \longrightarrow \coprod_{n=0}^{\infty} Y_{n} \longrightarrow Z,
$$

with $Y_{n}$ in $T$. Our problem is that the complexes $\Sigma W_{n}^{*}$ need not lie in $T$. They are bounded below, but the modules $\left\{W_{n}^{j}\right\}^{*}$ do not need to be finitely generated or projective. To complete the proof it therefore suffices to show that, for each $n$, the composite $C_{n} \longrightarrow \Sigma W_{n}^{*} \longrightarrow Z$ can also be expressed as $C_{n} \longrightarrow Y_{n} \longrightarrow Z$, with $Y_{n} \in T$.

Now observe that, for every $n \geq 0$, the composite $C_{n} \longrightarrow \Sigma W_{n}^{*} \longrightarrow Z$ is a chain map

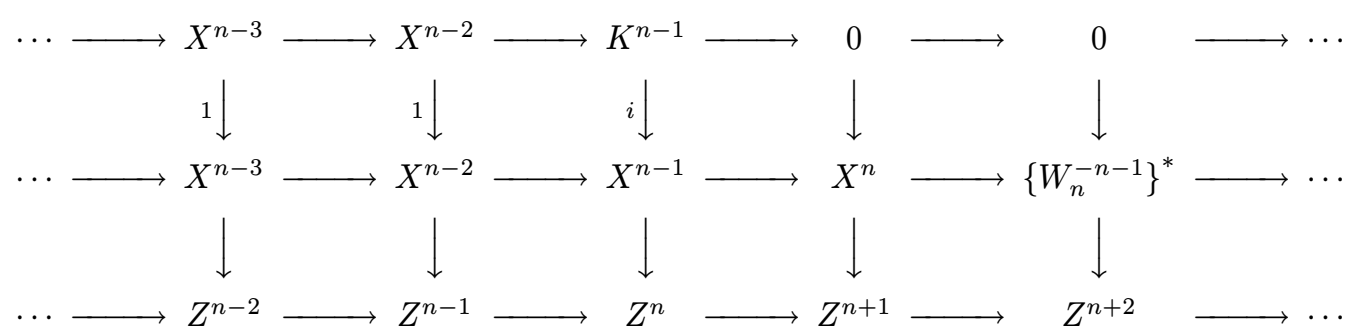

and this map is unaffected by what happens in degrees $\geq n+2$. In other words, if we leave the diagram unchanged in degrees $<n+2$, then the composite above will be equal to any possible composite below

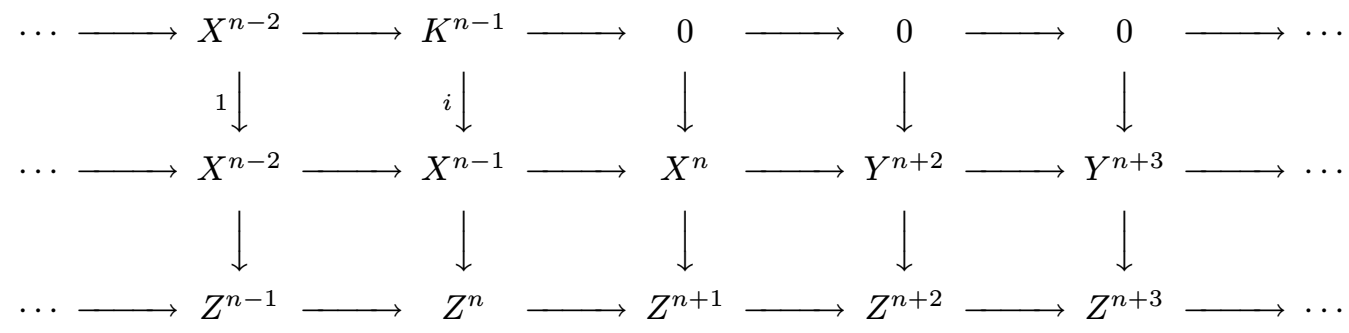

To complete the proof, it suffices therefore to show that

(††) We can complete the commutative diagram

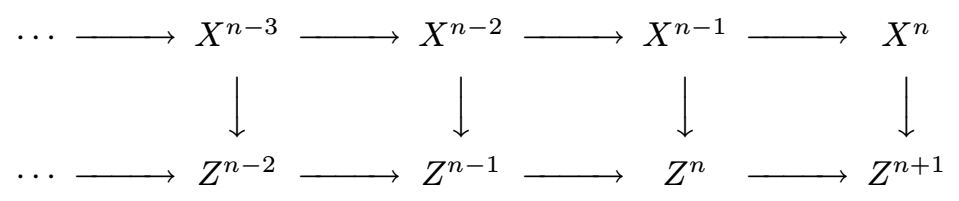

to a chain map

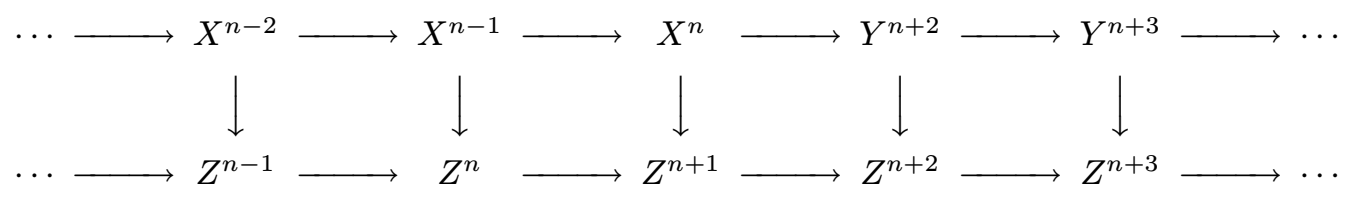

with the $Y^{j}$ finitely generated and projective.

The remainder of the proof will establish $(\dagger \dagger)$. 
As in the proof of [12, Lemma 4.1], we proceed inductively. Suppose we have the chain map defined as far as

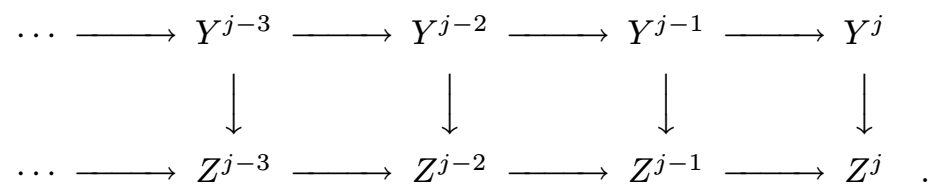

From the diagram

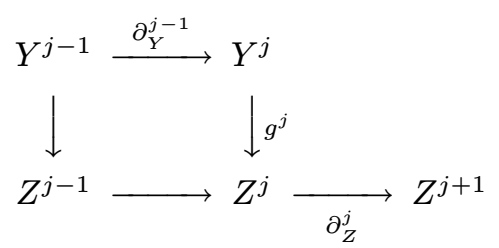

we note that $\partial_{Z}^{j} g^{j}: Y^{j} \longrightarrow Z^{j+1}$ is a map from the finitely generated, projective module $Y^{j}$ to the flat module $Z^{j+1}$, and that the composite

$$
Y^{j-1} \stackrel{\partial_{Y}^{j-1}}{\longrightarrow} Y^{j} \stackrel{\partial_{Z}^{j} g^{j}}{\longrightarrow} Z^{j+1}
$$

vanishes. From [12, Corollary 3.3] we deduce that the map $\partial_{Z}^{j} g^{j}: Y^{j} \longrightarrow Z^{j+1}$ factors as

$$
Y^{j} \stackrel{\partial_{Y}^{j}}{\longrightarrow} Y^{j+1} \stackrel{g^{j+1}}{\longrightarrow} Z^{j+1},
$$

with $Y^{j+1}$ finitely generated and projective, and so that the composite

$$
Y^{j-1} \stackrel{\partial_{Y}^{j-1}}{\longrightarrow} Y^{j} \stackrel{\partial_{Y}^{j}}{\longrightarrow} Y^{j+1}
$$

vanishes. This precisely means that we have extended the chain map to

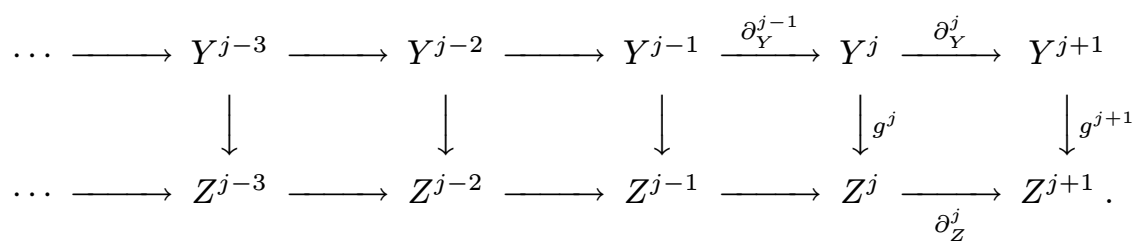

This completes the inductive step.

Lemma 1.9. - Suppose we are given two composable tensor-phantom morphisms

$$
X \stackrel{f}{\longrightarrow} Y \stackrel{g}{\longrightarrow} Z \text {. }
$$

Suppose furthermore that $X$ belongs to the set $T$, of $[12$, Construction 4.3]. Then the composite $g f: X \longrightarrow Z$ vanishes in $\mathbf{K}(R$-Flat).

Proof. - By Lemma 1.8 we know that $f$ must factor as

$$
X \longrightarrow \coprod_{n=0}^{\infty} C_{n} \stackrel{\coprod_{n=0}^{\infty} f_{n}}{\longrightarrow} \coprod_{n=0}^{\infty} Y_{n} \longrightarrow Y
$$

where the $C_{n}$ are bounded complexes and the $Y_{n}$ belong to $T$. Because $C_{n}$ is bounded, we may choose integers $N(n)$, so that $C_{n}^{i}=0$ if $i \geq N(n)$. Now the composite $Y_{n} \longrightarrow Y \stackrel{g}{\longrightarrow} Z$ 
must be a tensor-phantom map, because $g$ is. Being a tensor-phantom map from an object $Y_{n} \in T$ to the object $Z \in \mathbf{K}(R$-Flat $)$, it must, by Lemma 1.6, factor as

$$
Y_{n} \longrightarrow Z_{n} \longrightarrow Z,
$$

with $Z_{n}^{i}=0$ if $i \leq N(n)$. The composite

$$
C_{n} \longrightarrow Y_{n} \longrightarrow Z_{n}
$$

is a map from the complex $C_{n}$ to the complex $Z_{n}$, and $C_{n}^{i}=0$ if $i \geq N(n)$, while $Z_{n}^{i}=0$ if $i \leq N(n)$. The composite must therefore vanish. This means that the longer composite

$$
X \longrightarrow \coprod_{n=0}^{\infty} C_{n} \longrightarrow \coprod_{n=0}^{\infty} Y_{n} \longrightarrow \coprod_{n=0}^{\infty} Z_{n} \longrightarrow Z
$$

must also vanish, but this is $g f: X \longrightarrow Z$.

\section{The relation with $\mathscr{S}$-local objects}

In $\S 1$ we studied the formal properties of tensor-phantom maps. In this section we plan to disclose why we care about them. We begin by reminding the reader of bland generalities.

REMINDER 2.1. - We have an inclusion of triangulated categories $i_{*}: \mathscr{S} \longrightarrow \mathscr{T}$; in our case $\mathscr{T}$ happens to be $\mathbf{K}(R$-Flat $)$, while $\mathscr{S} \subset \mathscr{T}$ is $\mathbf{K}(R \text {-Proj })^{\perp} \subset \mathbf{K}(R$-Flat $)$. We want to produce objects in $\mathscr{S}^{\perp}$ and prove that they cogenerate. We recall the terminology of [11, Definition 9.1.3]; the objects of $\mathscr{S}^{\perp}$ are usually called $\mathscr{S}$-local objects. The next lemma will produce some $\mathscr{S}$-local objects, but in the larger category $\mathbf{K}(R-$ Mod $)$ rather than in $\mathbf{K}(R-$ Flat $) \subset \mathbf{K}(R-$ Mod $)$.

Lemma 2.2. - Let I be any complex of right $R$-modules. Then $\mathscr{H}$ om $m_{\mathbb{Z}}(I, \mathbb{Q} / \mathbb{Z})$ is a chain complex of left $R$-modules, and is $\mathscr{S}$-local.

Proof. - Let $X$ be any complex in $\mathscr{S}$. By [12, Proposition 9.1] the chain complex $I \otimes_{R} X$ is acyclic. Since $\mathbb{Q} / \mathbb{Z}$ is an injective abelian group, the complex

$$
\mathscr{H} o m_{\mathbb{Z}}\left(I \otimes_{R} X, \mathbb{Q} / \mathbb{Z}\right)
$$

is also acyclic. But

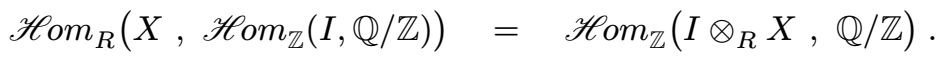

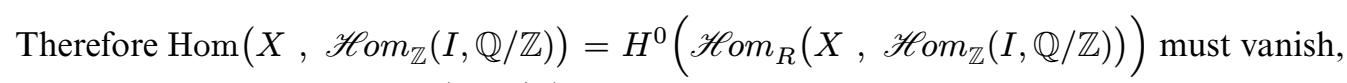
and we conclude that $\mathscr{H} o m_{\mathbb{Z}}(I, \mathbb{Q} / \mathbb{Z})$ is $\mathscr{S}$-local.

REMARK 2.3. - This means that, in the category $\mathbf{K}(R$-Mod), we have no difficulty at all in producing $\mathscr{S}$-local objects. Our problem is that we are after $\mathscr{S}$-local objects in $\mathbf{K}(R$-Flat $)$. If $I$ is an arbitrary chain complex of right $R$-modules, then the modules in the chain complex $\mathscr{H} o m_{\mathbb{Z}}(I, \mathbb{Q} / \mathbb{Z})$ are under no obligation to be flat. 
REMARK 2.4. - What we are about to say is an aside, which the reader can safely skip.

In Lemma 2.2 we produced a great many objects of $\mathbf{K}(R-$ Mod $)$, all of which are $\mathscr{S}$-local. It is conceivable that they cogenerate the orthogonal of $\mathscr{S}$ in $\mathbf{K}(R-$ Mod $)$; I have no idea. It is possible that, by using them, one might be able to give yet another proof that the inclusion $\mathscr{S} \longrightarrow \mathbf{K}(R-$ Mod $)$ has a right adjoint. Of course, [14, Theorem 3.1] already told us that the adjoint exists, and by now there are other proofs as well.

REMARK 2.5. - Remark 2.3 is something of a digression. In this article we need $\mathscr{S}$-local objects in $\mathbf{K}(R-$ Flat $)$, not $\mathbf{K}(R-$ Mod $)$.

Of course Remark 2.3 observed that, in the category $\mathbf{K}(R-\mathrm{Mod})$, it is easy to construct $\mathscr{S}$-local objects. From [14, Theorem 3.2] we know that the inclusion $\mathbf{K}(R$-Flat $) \longrightarrow$ $\mathbf{K}(R-$ Mod $)$ has a right adjoint $J: \mathbf{K}(R-$ Mod $) \longrightarrow \mathbf{K}(R$-Flat $)$. Next we note the trivial fact:

Lemma 2.6. - Let $X$ be an $\mathscr{S}$-local object in $\mathbf{K}(R-$ Mod $)$. Let $J: \mathbf{K}(R-\mathrm{Mod}) \longrightarrow$ $\mathbf{K}(R-$ Flat $)$ be a right adjoint to the inclusion $\mathbf{K}(R-$ Flat $) \longrightarrow \mathbf{K}(R-$ Mod $)$. Then $J X$ is an $\mathscr{S}$-local object in $\mathbf{K}(R$-Flat).

Remark 2.7. - Lemma 2.2 tells us that $\mathscr{H} o m(I, \mathbb{Q} / \mathbb{Z})$ is an $\mathscr{S}$-local object of $\mathbf{K}(R$-Mod $)$, while Lemma 2.6 establishes that $J(\mathscr{H} o m(I, \mathbb{Q} / \mathbb{Z}))$ is an $\mathscr{S}$-local object of $\mathbf{K}(R$-Flat $)$. In Theorem 4.7 we will show that a suitable set of objects of the form $J(\mathscr{H}$ om $(I, \mathbb{Q} / \mathbb{Z}))$ cogenerates $\mathscr{S}^{\perp}$.

Now it is high time to reveal what all of this has to do with tensor-phantom maps.

Lemma 2.8. - Let $J: \mathbf{K}(R-$ Mod $) \longrightarrow \mathbf{K}(R-$ Flat $)$ be a right adjoint to the inclusion $\mathbf{K}(R$-Flat $) \longrightarrow \mathbf{K}(R-$ Mod $)$. A map $f: Y \longrightarrow Z$ in $\mathbf{K}(R$-Flat $)$ is tensor-phantom if and only if, for every test-complex $I$ and for every map $g: Z \longrightarrow J\left(\mathscr{H}_{0} m_{\mathbb{Z}}(I, \mathbb{Q} / \mathbb{Z})\right)$, the composite

$$
Y \stackrel{f}{\longrightarrow} Z \stackrel{g}{\longrightarrow} J\left(\mathscr{H}_{\mathrm{om}}(I, \mathbb{Q} / \mathbb{Z})\right)
$$

is null homotopic.

Proof. - By definition the map $f: Y \longrightarrow Z$ is tensor-phantom if and only if, for every test-complex $I$, the map

$$
I \otimes_{R} Y \stackrel{1 \otimes f}{\longrightarrow} I \otimes_{R} Z
$$

vanishes in cohomology. If $I$ is a test-complex, then so is any suspension $\Sigma^{n} I$. Hence $f: Y \longrightarrow Z$ is tensor-phantom if and only if, for every test-complex $I$, the map

$$
H^{0}\left(I \otimes_{R} Y\right) \stackrel{H^{0}(1 \otimes f)}{\longrightarrow} H^{0}\left(I \otimes_{R} Z\right)
$$

vanishes. Since $\mathbb{Q} / \mathbb{Z}$ is an injective cogenerator in the category of abelian groups, it is equivalent for the map

$$
\operatorname{Hom}_{\mathbb{Z}}\left(H^{0}\left(I \otimes_{R} Z\right), \mathbb{Q} / \mathbb{Z}\right) \longrightarrow \operatorname{Hom}_{\mathbb{Z}}\left(H^{0}\left(I \otimes_{R} Y\right), \mathbb{Q} / \mathbb{Z}\right)
$$

to vanish, for every test-complex $I$. This map identifies as

$$
\operatorname{Hom}_{\mathbf{K}(\mathbb{Z})}\left(I \otimes_{R} Z, \mathbb{Q} / \mathbb{Z}\right) \longrightarrow \operatorname{Hom}_{\mathbf{K}(\mathbb{Z})}\left(I \otimes_{R} Y, \mathbb{Q} / \mathbb{Z}\right) .
$$


But this, in turn, identifies with the map

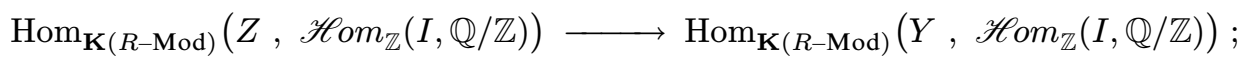

to say that all these maps vanish is to say that any chain map $Z \longrightarrow \mathscr{H}_{0} m_{\mathbb{Z}}(I, \mathbb{Q} / \mathbb{Z})$ composes with $f: Y \longrightarrow Z$ to give a null homotopic map.

Finally note that $Y$ and $Z$ are objects in $\mathbf{K}(R-$ Flat $)$. The inclusion of $\mathbf{K}(R$-Flat $)$ into $\mathbf{K}(R-\mathrm{Mod})$ has a right adjoint $J$. The composites

$$
Y \stackrel{f}{\longrightarrow} Z \longrightarrow \mathscr{H} \mathrm{om} \mathrm{Z}_{\mathbb{Z}}(I, \mathbb{Q} / \mathbb{Z})
$$

will all vanish if and only if the composites

$$
Y \stackrel{f}{\longrightarrow} Z \longrightarrow J\left(\mathscr{H}_{\mathrm{om}} \mathrm{m}_{\mathbb{Z}}(I, \mathbb{Q} / \mathbb{Z})\right)
$$

are null.

\section{A reminder of right coherent rings}

In Remark 2.7 we observed that the complexes $J\left(\mathscr{H} o m_{\mathbb{Z}}(I, \mathbb{Q} / \mathbb{Z})\right)$ are $\mathscr{S}$-local objects in $\mathbf{K}(R$-Flat $)$. In this section we will note that, as long as $I$ is a complex of injective right $R$-modules over a right coherent ring $R$, then $\mathscr{H} o m_{\mathbb{Z}}(I, \mathbb{Q} / \mathbb{Z})$ is a complex of flat $R$-modules. There is no need to apply the functor $J$.

This section reminds the reader of the statements and proofs of a couple of basic facts about right coherent rings. I do this partly to keep the paper self-contained, but mostly to highlight where the right coherence of $R$ is being used.

If $I$ is a right $R$-module, then $\operatorname{Hom}_{\mathbb{Z}}(I, \mathbb{Q} / \mathbb{Z})$ is a left $R$-module. If $F$ is another right $R$-module, composition gives a map

$$
F \otimes \operatorname{Hom}_{R}(F, I) \otimes \operatorname{Hom}_{\mathbb{Z}}(I, \mathbb{Q} / \mathbb{Z}) \longrightarrow \mathbb{Q} / \mathbb{Z},
$$

which yields a natural map

$$
F \otimes_{R} \operatorname{Hom}_{\mathbb{Z}}(I, \mathbb{Q} / \mathbb{Z}) \longrightarrow \operatorname{Hom}_{\mathbb{Z}}\left(\operatorname{Hom}_{R}(F, I), \mathbb{Q} / \mathbb{Z}\right) .
$$

This map is obviously an isomorphism when $F=R$ is free of rank 1 . Because it commutes with finite direct sums in $F$, the map is an isomorphism whenever $F$ is a free module of finite rank. Now we note

Lemma 3.1. - Let $R$ be a right coherent ring, and let $I$ be an injective right $R$-module. Then the left $R$-module $\operatorname{Hom}(I, \mathbb{Q} / \mathbb{Z})$ is flat.

Proof. - It suffices to show that, for each finitely presented right $R$-module $M$, the group $\operatorname{Tor}_{1}^{R}(M, \operatorname{Hom}(I, \mathbb{Q} / \mathbb{Z}))$ vanishes. The ring $R$ is assumed right coherent, and $M$ is a finitely presented right $R$-module. We may choose a resolution for $M$

$$
P_{2} \longrightarrow P_{1} \longrightarrow P_{0} \longrightarrow M \longrightarrow 0
$$

with the $P_{2}, P_{1}$ and $P_{0}$ finitely generated, free right $R$-modules. We need to show the exactness of the sequence

$$
P_{2} \otimes_{R} \operatorname{Hom}(I, \mathbb{Q} / \mathbb{Z}) \longrightarrow P_{1} \otimes_{R} \operatorname{Hom}(I, \mathbb{Q} / \mathbb{Z}) \longrightarrow P_{0} \otimes_{R} \operatorname{Hom}(I, \mathbb{Q} / \mathbb{Z}) .
$$

$4^{\text {e }}$ SÉRIE - TOME $44-2011-$ No $^{\circ}$ 
To this end observe the diagram

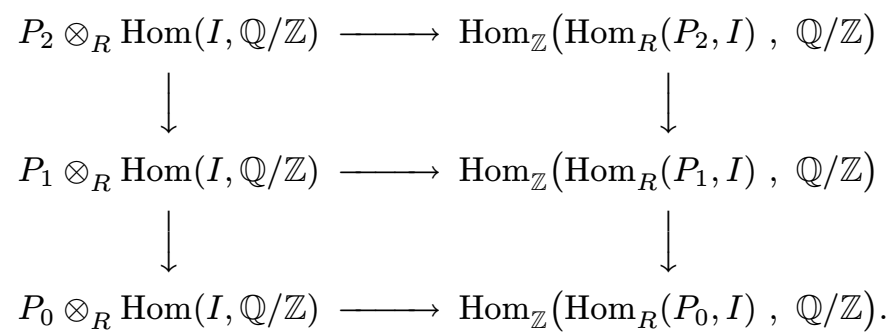

The horizontal maps are isomorphisms, and we want to show the left column exact. It suffices, therefore, to show the right column exact. But now the fact that $I$ is an injective right $R$-module says that

$$
\operatorname{Hom}_{R}\left(P_{0}, I\right) \longrightarrow \operatorname{Hom}_{R}\left(P_{1}, I\right) \longrightarrow \operatorname{Hom}_{R}\left(P_{2}, I\right)
$$

is an exact sequence of abelian groups, while the fact that $\mathbb{Q} / \mathbb{Z}$ is an injective abelian group gives the desired exactness of the right column.

An immediate consequence is

Lemma 3.2. - Let $R$ be a right coherent ring. If I is a complex of injective right $R$-modules, then $X=\mathscr{H}$ om $(I, \mathbb{Q} / \mathbb{Z})$ is a complex of flat left $R$-modules. Furthermore, $X$ is orthogonal of $\mathscr{S}$.

Proof. - The fact that $X$ is a chain complex of flat $R$-modules may be found in Lemma 3.1, and the orthogonality to $\mathscr{S}$ comes from Lemma 2.2.

Before we leave this section, we want to note a fact about products of flat modules.

Lemma 3.3. - Let $R$ be a right coherent ring. Suppose that $\left\{F_{\lambda}, \lambda \in \Lambda\right\}$ is a set of flat left $R$-modules. Then the product

$$
\prod_{\lambda \in \Lambda} F_{\lambda}
$$

is a flat left R-module.

Proof. - As in the proof of Lemma 3.1 it suffices to prove that, given an exact sequence

$$
P_{2} \longrightarrow P_{1} \longrightarrow P_{0}
$$

of free right $R$-modules of finite rank, the sequence

$$
P_{2} \otimes_{R}\left\{\prod_{\lambda \in \Lambda} F_{\lambda}\right\} \longrightarrow P_{1} \otimes_{R}\left\{\prod_{\lambda \in \Lambda} F_{\lambda}\right\} \longrightarrow P_{0} \otimes_{R}\left\{\prod_{\lambda \in \Lambda} F_{\lambda}\right\}
$$

is also exact. Now for a free module of finite rank, the natural map

$$
P \otimes_{R}\left\{\prod_{\lambda \in \Lambda} F_{\lambda}\right\} \longrightarrow \prod_{\lambda \in \Lambda}\left\{P \otimes_{R} F_{\lambda}\right\}
$$

is an isomorphism, so the sequence above becomes

$$
\prod_{\lambda \in \Lambda}\left\{P_{2} \otimes_{R} F_{\lambda}\right\} \longrightarrow \prod_{\lambda \in \Lambda}\left\{P_{1} \otimes_{R} F_{\lambda}\right\} \longrightarrow \prod_{\lambda \in \Lambda}\left\{P_{0} \otimes_{R} F_{\lambda}\right\}
$$


which is obviously exact.

\section{There are many $\mathscr{S}$-local objects}

In this section and the next we will show how to build up more $\mathscr{S}$-local objects. In the case where the ring $R$ is right coherent the construction is elementary. In the general case it uses the functor $J: \mathbf{K}(R-\mathrm{Mod}) \longrightarrow \mathbf{K}(R-$ Flat $)$, which is right adjoint to the inclusion $\mathbf{K}(R-$ Flat $) \longrightarrow \mathbf{K}(R-$ Mod $)$.

REMinder 4.1. - If $I$ is any chain complex of right $R$-modules, then Lemma 2.2 says that the complex $\mathscr{H} o m_{\mathbb{Z}}(I, \mathbb{Q} / \mathbb{Z})$ is an $\mathscr{S}$-local object in $\mathbf{K}(R$-Mod). From Lemma 2.6 it follows that $J\left(\mathscr{H} o m_{\mathbb{Z}}(I, \mathbb{Q} / \mathbb{Z})\right)$ is an $\mathscr{S}$-local object in $\mathbf{K}(R$-Flat $)$. In the special case, where $R$ happens to be right coherent and $I$ is a complex of injective right $R$-modules, Lemma 3.2 tells us that $\mathscr{H} o m_{\mathbb{Z}}(I, \mathbb{Q} / \mathbb{Z})$ is a chain complex of flat modules as it stands. For right coherent rings $R$, there is no need to apply the functor $J$.

Remark 4.2. - From [14, Theorem 3.2] we know that the inclusion $\mathbf{K}(R$-Flat $) \longrightarrow$ $\mathbf{K}(R-$ Mod $)$ has a right adjoint $J: \mathbf{K}(R-$ Mod $) \longrightarrow \mathbf{K}(R$-Flat $)$. This means that the category $\mathbf{K}(R$-Flat $)$ has products; given a collection $\left\{Z_{\lambda}, \lambda \in \Lambda\right\}$ of objects in $\mathbf{K}(R$-Flat), the product in $\mathbf{K}(R-$ Flat $)$ is obtained by forming the product in $\mathbf{K}(R-\mathrm{Mod})$, and then applying the functor $J$. In symbols, the product in the category $\mathbf{K}(R-$ Flat $)$ is

$$
J\left(\prod_{\lambda \in \Lambda} Z_{\lambda}\right) .
$$

If $R$ happens to be right coherent, then products of flat modules are flat by Lemma 3.3. For right coherent rings there is no need to apply $J$ to the product.

It is time to get to work.

Construction 4.3. - Let us choose a set $\Lambda$ of representatives for the homotopy equivalence classes of test complexes; every test-complex $I$ is homotopy equivalent to one of $\left\{I_{\lambda}, \lambda \in \Lambda\right\}$. Remark 1.2 guarantees that this can be done. For each $\lambda \in \Lambda$, let

$$
F_{\lambda}=J\left(\mathscr{H} o m_{\mathbb{Z}}\left(I_{\lambda}, \mathbb{Q} / \mathbb{Z}\right)\right) .
$$

Let $\mathscr{F}$ be the colocalizing subcategory cogenerated by the objects $\left\{F_{\lambda}, \lambda \in \Lambda\right\}$. This means that $\mathscr{F}$ is the smallest triangulated subcategory of $\mathbf{K}(R$-Flat $)$, containing all the $F_{\lambda}$ and closed under products. Since $\mathscr{S}^{\perp}$ is colocalizing and contains all the objects $F_{\lambda}=J\left(\mathscr{H}\right.$ om $\left._{\mathbb{Z}}\left(I_{\lambda}, \mathbb{Q} / \mathbb{Z}\right)\right)$, it follows that $\mathscr{F} \subset \mathscr{S}^{\perp}$. The main fact we will prove, in what follows, is that $\mathscr{F}=\mathscr{S}^{\perp}$.

Lemma 4.4. - Let $Z$ be an object of $\mathbf{K}(R$-Flat). There exists a distinguished triangle in $\mathbf{K}(R-$ Flat $)$

$$
Y \stackrel{f}{\longrightarrow} Z \longrightarrow F \longrightarrow \Sigma Y,
$$

so that $f: Y \longrightarrow Z$ is a tensor-phantom map, and $F \in \mathscr{F}$.

$4^{\mathrm{e}}$ SÉRIE - TOME $44-2011-\mathrm{N}^{\circ} 4$ 
Proof. - Let $F$ be the product of $F_{\lambda}$, over all morphisms $Z \longrightarrow F_{\lambda}$, for any $\lambda \in \Lambda$. Let $Z \longrightarrow F$ be the natural map. Complete it to a triangle

$$
Y \stackrel{f}{\longrightarrow} Z \longrightarrow F \longrightarrow \Sigma Y \text {. }
$$

Clearly $F \in \mathscr{F}$, since it is a product of $F_{\lambda}$ 's. By Construction 4.3 every test complex $I$ is homotopy equivalent to an $I_{\lambda}$, and hence every map $Z \longrightarrow J\left(\mathscr{H} o m_{\mathbb{Z}}(I, \mathbb{Q} / \mathbb{Z})\right) \cong F_{\lambda}$ must factor through $Z \longrightarrow F$. The triangle $Y \longrightarrow Z \longrightarrow F \longrightarrow \Sigma Y$ tells us that every composite

$$
Y \stackrel{f}{\longrightarrow} Z \longrightarrow J\left(\mathscr{H}_{\mathrm{om}}(I, \mathbb{Q} / \mathbb{Z})\right)
$$

must vanish. Lemma 2.8 permits us to conclude that $f$ is tensor-phantom.

Lemma 4.5. - Let $T$ be the set of objects in $\mathbf{K}(R-$ Proj), given in [12, Construction 4.3] or in Reminder 1.5. Every object $Z \in \mathbf{K}(R$-Flat $)$ admits a map $Z \longrightarrow F$, where $F \in \mathscr{F}$, and so that, for all objects $t \in T$, the map

$$
\operatorname{Hom}_{\mathbf{K}(R-\mathrm{Flat})}(t, Z) \longrightarrow \operatorname{Hom}_{\mathbf{K}(R-\mathrm{Flat})}(t, F)
$$

is injective.

Proof. - By Lemma 4.4 there exists a triangle in $\mathbf{K}(R$-Flat $)$

$$
Y \stackrel{f}{\longrightarrow} Z \longrightarrow F_{1} \longrightarrow \Sigma Y,
$$

with $F_{1} \in \mathscr{F}$, and with $f$ a tensor-phantom map. Applying Lemma 4.4 again, this time to the object $Y$, there exists a triangle

$$
X \stackrel{g}{\longrightarrow} Y \longrightarrow F_{2} \longrightarrow \Sigma X,
$$

with $F_{2} \in \mathscr{F}$, and with $g$ a tensor-phantom map. Now we build an octahedron from the two composable maps $X \stackrel{g}{\longrightarrow} Y \stackrel{f}{\longrightarrow} Z$. We obtain two distinguished triangles

$$
\begin{aligned}
& X \longrightarrow F\left(\longrightarrow F \longrightarrow F \longrightarrow F_{1},\right. \\
& F_{2} \longrightarrow F F_{2} .
\end{aligned}
$$

The second of these tells us that $F$ must be an object of $\mathscr{F}$. In the first of these two triangles, the map $f g: X \longrightarrow Z$ is a composite of two tensor-phantom maps. Lemma 1.9 tells us that, if $t$ is any object in $T$, then any composite

$$
t \longrightarrow X \stackrel{g}{\longrightarrow} Y \stackrel{f}{\longrightarrow} Z
$$

must vanish. In the exact sequence

$$
\operatorname{Hom}(t, X) \stackrel{\operatorname{Hom}(t, f g)}{\longrightarrow} \operatorname{Hom}(t, Z) \longrightarrow \operatorname{Hom}(t, F)
$$

we know that the map $\operatorname{Hom}(t, f g)$ vanishes. Hence the map $\operatorname{Hom}(t, Z) \longrightarrow \operatorname{Hom}(t, F)$ must be injective.

Lemma 4.6. - Let $X \longrightarrow F$ be a morphism in $\mathbf{K}(R$-Flat), and suppose $F \in \mathscr{F}$. Then there is a factorization

$$
X \longrightarrow F^{\prime} \longrightarrow F
$$

with $F^{\prime} \in \mathscr{F}$ and so that, for all $t \in T$, the two maps

$$
\operatorname{Hom}(t, X) \longrightarrow \operatorname{Hom}(t, F) \quad \text { and } \operatorname{Hom}\left(t, F^{\prime}\right) \longrightarrow \operatorname{Hom}(t, F)
$$

have the same image $I \subset \operatorname{Hom}(t, F)$. 
Proof. - Complete $X \longrightarrow F$ to a triangle

$$
X \longrightarrow F \longrightarrow Y \longrightarrow \Sigma X \text {. }
$$

By Lemma 4.5 there exists a morphism $Y \longrightarrow F^{\prime \prime}$, with $F^{\prime \prime} \in \mathscr{F}$ and so that $\operatorname{Hom}(t, Y) \longrightarrow$ $\operatorname{Hom}\left(t, F^{\prime \prime}\right)$ is injective. The triangle gives us an exact sequence

$$
\operatorname{Hom}(t, X) \longrightarrow \operatorname{Hom}(t, F) \longrightarrow \operatorname{Hom}(t, Y),
$$

and the injectivity of $\operatorname{Hom}(t, Y) \longrightarrow \operatorname{Hom}\left(t, F^{\prime \prime}\right)$ means that the sequence

$$
\operatorname{Hom}(t, X) \longrightarrow \operatorname{Hom}(t, F) \longrightarrow \operatorname{Hom}\left(t, F^{\prime \prime}\right)
$$

is also exact. Now form the distiniguished triangle

$$
F^{\prime} \longrightarrow F \longrightarrow F^{\prime \prime} \longrightarrow \Sigma F^{\prime} \text {. }
$$

Since $F, F^{\prime \prime}$ lie in $\mathscr{F}$, so does $F^{\prime}$. Because the composite $X \longrightarrow F \longrightarrow Y \longrightarrow F^{\prime \prime}$ clearly vanishes, it follows that the map $X \longrightarrow F$ must factor as $X \longrightarrow F^{\prime} \longrightarrow F$. We have a commutative diagram, where the rows are exact

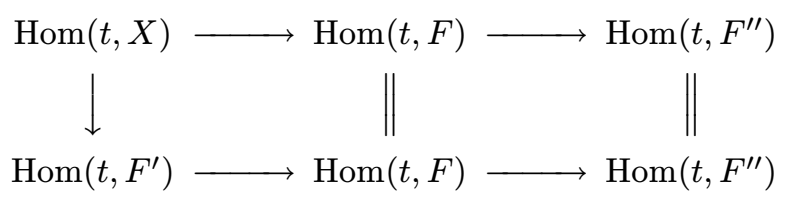

from which we conclude that the images of the maps

$$
\operatorname{Hom}(t, X) \longrightarrow \operatorname{Hom}(t, F) \quad \text { and } \operatorname{Hom}\left(t, F^{\prime}\right) \longrightarrow \operatorname{Hom}(t, F)
$$

must agree; both are equal to the kernel of $\operatorname{Hom}(t, F) \longrightarrow \operatorname{Hom}\left(t, F^{\prime \prime}\right)$.

Now we are ready for the main theorem; the second assertion is known now by several proofs, but we include it anyway since it drops out formally in the proof of the (new) first assertion.

THEOREM 4.7. - Let $R$ be any ring. Then $\mathscr{F}=\mathscr{S}^{\perp}=\left\{\mathbf{K}(R-\text { Proj })^{\perp}\right\}^{\perp}$, and furthermore there is a right adjoint to the inclusion $\mathscr{S}=\mathbf{K}(R-\text { Proj })^{\perp} \longrightarrow \mathbf{K}(R-$ Flat $)$.

Proof. - Let $Y$ be any object of $\mathbf{K}(R$-Flat $)$. Lemma 4.5 tells us that there exists a morphism $Y \longrightarrow F_{0}$, with $F_{0} \in \mathscr{F}$, and so that, for all $t \in T$, the map $\operatorname{Hom}(t, Y) \longrightarrow$ $\operatorname{Hom}\left(t, F_{0}\right)$ is injective. Now we inductively construct a sequence of morphisms in $\mathscr{F}$

$$
\cdots \longrightarrow F_{3} \longrightarrow F_{2} \longrightarrow F_{1} \longrightarrow F_{0},
$$

and a map from $Y$ to the sequence. The construction is as in Lemma 4.6; we factor the map $Y \longrightarrow F_{n}$ as $Y \longrightarrow F_{n+1} \longrightarrow F_{n}$, so that the images of

$$
\operatorname{Hom}(t, Y) \longrightarrow \operatorname{Hom}\left(t, F_{n}\right) \quad \text { and } \operatorname{Hom}\left(t, F_{n+1}\right) \longrightarrow \operatorname{Hom}\left(t, F_{n}\right)
$$

agree. For every object $t \in T$, the sequence

$$
\cdots \longrightarrow \operatorname{Hom}\left(t, F_{3}\right) \longrightarrow \operatorname{Hom}\left(t, F_{2}\right) \longrightarrow \operatorname{Hom}\left(t, F_{1}\right)
$$

identifies as the direct sum of two sequences

$$
\begin{array}{lllll}
\cdots \stackrel{1}{\longrightarrow} \operatorname{Hom}(t, Y) \stackrel{1}{\longrightarrow} \operatorname{Hom}(t, Y) \stackrel{1}{\longrightarrow} \operatorname{Hom}(t, Y) \\
\cdots \stackrel{0}{\longrightarrow} K_{3} \stackrel{0}{\longrightarrow} K_{2} \stackrel{0}{\longrightarrow} K_{1} .
\end{array}
$$

$4^{\text {e }}$ SÉRIE - TOME $44-2011-$ No $^{\circ}$ 
Now let $F$ be the homotopy limit of the sequence $F_{n}$; that is, it fits in a triangle

$$
F \longrightarrow \prod_{n=1}^{\infty} F_{n} \stackrel{1-\text { shift }}{\longrightarrow} \prod_{n=1}^{\infty} F_{n} \longrightarrow \Sigma F \text {. }
$$

Since each of the $F_{n}$ lies in $\mathscr{F}$, so does the homotopy limit $F$. The map $Y \longrightarrow \prod_{n=1}^{\infty} F_{n}$ factors through $F$, and if we apply the functor $\operatorname{Hom}(t,-)$ to the triangle, with $t \in T$, we easily see that $\operatorname{Hom}(t, Y) \longrightarrow \operatorname{Hom}(t, F)$ is an isomorphism. Now complete $Y \longrightarrow F$ to a triangle

$$
S \longrightarrow Y \longrightarrow F \longrightarrow \Sigma S \text {. }
$$

We have that $F \in \mathscr{F} \subset \mathscr{S}^{\perp}$. The fact that $\operatorname{Hom}(t, Y) \longrightarrow \operatorname{Hom}(t, F)$ is an isomorphism tells us that $\operatorname{Hom}(t, S)$ vanishes, for every $t \in T$. This makes $S$ orthogonal to the category generated by $T$; [12, Proposition 7.4] tells us that $T$ generates $\mathbf{K}(R-\operatorname{Proj})$. Thus $S \in \mathbf{K}(R-\text { Proj })^{\perp}=\mathscr{S}$.

Given an object $Y \in \mathbf{K}(R-$ Flat $)$, we have exhibited a distinguished triangle

$$
S \longrightarrow Y \longrightarrow F \longrightarrow \Sigma S
$$

with $S \in \mathscr{S}$ and $F=\mathscr{S}^{\perp}$. From [11, Theorem 9.1.13] we deduce that the inclusion $i_{*}: \mathscr{S} \longrightarrow \mathbf{K}(R$-Flat $)$ has a right adjoint. If $Y \in \mathscr{S}^{\perp}$, the distinguished triangle

$$
S \longrightarrow Y \longrightarrow F \longrightarrow \Sigma S
$$

is such that both $Y$ and $F$ lie in $\mathscr{S}^{\perp}$, and hence so does $S$. Therefore $S$ belongs both to $\mathscr{S}$ and to $\mathscr{S}^{\perp}$, and must vanish. It follows that $Y \longrightarrow F$ is an isomorphism; we conclude that $\mathscr{F}=\mathscr{S}^{\perp}$.

\section{The special case of compactly generated $\mathbf{K}(R-$ Flat $)$}

In Theorem 4.7 we proved, for any ring $R$, that the subcategory $\mathscr{S}^{\perp} \subset \mathbf{K}(R$-Flat $)$ is equal to the subcategory $\mathscr{F} \subset \mathbf{K}(R-$ Flat $)$, the colocalizing subcategory cogenerated by the objects $J\left(\mathscr{H} o m_{\mathbb{Z}}(I, \mathbb{Q} / \mathbb{Z})\right)$.

The statement that the objects $J\left(\mathscr{H} o m_{\mathbb{Z}}(I, \mathbb{Q} / \mathbb{Z})\right)$ cogenerate $\mathscr{S}^{\perp}$ is true for any ring $R$. In the general case I do not have an easier argument than the one given in the previous sections. If we are willing to restrict ourselves to the case where the ring $R$ is right coherent, then we have already observed that there is no need to use the functor $J$. What we will note in this section is that the entire proof simplifies, because the category $\mathbf{K}(R-\mathrm{Proj})$ is compactly generated; see [12, Proposition 7.14].

For the rest of this article $R$ is assumed to be a right coherent ring.

REMINDER 5.1. - We begin by reminding the reader of formal generalities. Let $\mathscr{T}$ be a triangulated category and let $\mathscr{S} \subset \mathscr{T}$ be a thick subcategory. Let $\mathscr{S}^{\perp}$ be the full subcategory of all $\mathscr{S}$-local objects in $\mathscr{T}$. Let $\pi: \mathscr{T} \longrightarrow \mathscr{T} / \mathscr{S}$ be the natural projection to the Verdier quotient. From [11, Lemma 9.1.5] we know that, if $x$ is any object in $\mathscr{T}$ and $y \in \mathscr{S}^{\perp}$, then

$$
\operatorname{Hom}_{\mathscr{T}}(x, y)=\operatorname{Hom}_{\mathscr{T} / \mathscr{S}}(\pi x, \pi y) .
$$


If we let $x$ and $y$ both be in the subcategory $\mathscr{S}^{\perp}$, we learn that the composite functor

$$
\mathscr{S}^{\perp} \stackrel{\text { inclusion }}{\longrightarrow} \mathscr{T} \stackrel{\pi}{\longrightarrow} \mathscr{T} / \mathscr{S}
$$

must be fully faithful. If the inclusion $i_{*}: \mathscr{S} \longrightarrow \mathscr{T}$ has a right adjoint, then [11, Theorem 9.1.16] asserts that the map $\mathscr{S}^{\perp} \longrightarrow \mathscr{T} / \mathscr{S}$ is an equivalence of categories.

We now consider two special cases: the first is the case where $\mathscr{T}=\mathbf{K}(R$-Flat $)$ and where $\mathscr{S}$ is the subcategory $\mathscr{S}=\mathbf{K}(R-\text { Proj })^{\perp}$ of [14, Notation 2.2]. A right adjoint to the inclusion $\mathscr{S} \longrightarrow \mathscr{T}$ exists by [14, Theorem 3.1], and hence the composite

$$
\mathscr{S}^{\perp} \stackrel{\text { inclusion }}{\longrightarrow} \mathscr{T} \stackrel{\pi}{\longrightarrow} \mathscr{T} / \mathscr{S}
$$

is an equivalence of categories. But we can also dualize and apply the general theory to the inclusion $\mathscr{S}^{\text {op }} \longrightarrow \mathscr{T}^{\text {op }}$; this inclusion also has a right adjoint, or equivalently the inclusion $\mathscr{S} \longrightarrow \mathscr{T}$ has a left adjoint. The existence of this adjoint can be seen as follows: from [12, Proposition 8.1] we know that the natural inclusion $j_{!}: \mathbf{K}(R-$ Proj $) \longrightarrow \mathbf{K}(R-$ Flat $)$ has a right adjoint, and formal nonsense, specifically [11, Corollary 9.1.14], tells us that the inclusion from $\mathscr{S}=\mathbf{K}(R \text {-Proj })^{\perp}$ into $\mathscr{T}=\mathbf{K}(R$-Flat $)$ has a left adjoint. By the dual of the argument in the previous paragraph we conclude that the composite

$$
\perp \mathscr{S} \stackrel{\text { inclusion }}{\longrightarrow} \mathscr{T} \stackrel{\pi}{\longrightarrow} \mathscr{T} / \mathscr{S}
$$

is also an equivalence of categories. But we know ${ }^{\perp} \mathscr{S}$; it is nothing other than $\mathbf{K}(R$-Proj), embedded into $\mathscr{T}=\mathbf{K}(R-$ Flat $)$ via the natural map. Thus we have equivalences

$$
\mathbf{K}(R-\text { Proj })={ }^{\perp} \mathscr{S} \cong \mathscr{T} / \mathscr{S} \cong \mathscr{S}^{\perp} \text {. }
$$

REMARK 5.2. - The reason for reminding the reader of these equivalences is that the compact generators for $\mathbf{K}(R$-Proj $)$, in their usual form, naturally lie in $\mathbf{K}(R$-Proj $)={ }^{\perp} \mathscr{S}$; see $\left[12\right.$, Proposition 7.12]. The cogenerators $\mathscr{H}_{0} m_{\mathbb{Z}}(I, \mathbb{Q} / \mathbb{Z})$ of this article belong naturally to the equivalent $\mathscr{S}^{\perp}$. If we want to compare the cogenerators of this article with the traditional ones that are produced from compact objects, then we need to keep in mind just how the equivalence ${ }^{\perp} \mathscr{S} \cong \mathscr{S}^{\perp}$ actually works.

Specifically, we will use the following. For any test-complex $I$ we have that $F=\mathscr{H}_{0} m_{\mathbb{Z}}(I, \mathbb{Q} / \mathbb{Z})$ belongs to $\mathscr{S}^{\perp}$. Choose any $X \in \mathbf{K}(R-$ Proj $)=\perp \mathscr{S}$; then we know that

$$
\operatorname{Hom}_{\mathbf{K}(R-\text { Flat }) / \mathscr{S}}(\pi X, \pi F)=\operatorname{Hom}_{\mathbf{K}(R-\text { Flat })}(X, F) .
$$

This by [11, Lemma 9.1.5], as discussed at the beginning of Reminder 5.1: we recalled that the natural map

$$
\operatorname{Hom}_{\mathscr{T}}(x, y) \longrightarrow \operatorname{Hom}_{\mathscr{T} / \mathscr{S}}(\pi x, \pi y)
$$

is an isomorphism if $y \in \mathscr{S}^{\perp}$ or (dually) if $x \in{ }^{\perp} \mathscr{S}$. Above we have a surfeit of reasons for this map to be an isomorphism, since we know both that $F \in \mathscr{S}^{\perp}$ and that $X \in{ }^{\perp} \mathscr{S}$.

Lemma 5.3. - Let $R$ be a ring, let I be a bounded below complex of right $R$-modules, and put $F=\mathscr{H}_{\mathrm{O}} \mathrm{m}_{\mathbb{Z}}(I, \mathbb{Q} / \mathbb{Z})$. Let $P$ be a chain complex in the set $T$ of $[12$, Construction 4.3] or Reminder 1.5. Then

$$
\operatorname{Hom}_{\mathbf{K}(R-\text { Mod })}(P, F)=\operatorname{Hom}_{K(\mathbb{Z})}\left(\mathscr{H} o m_{R}\left(P^{*}, I\right), \mathbb{Q} / \mathbb{Z}\right) .
$$

$4{ }^{\text {e }}$ SÉRIE - TOME $44-2011-\mathrm{N}^{\circ} 4$ 
Proof. - We should perhaps remind the reader; the objects in the set $T$ are bounded below chain complexes of finitely generated, projective left $R$-modules, and the complex $P^{*}$ is the complex $\mathscr{H} o m(P, R)$; it is a bounded above complex of finitely generated, projective right $R$-modules.

Now for the proof. We recall that $F=\mathscr{H} o m_{\mathbb{Z}}(I, \mathbb{Q} / \mathbb{Z})$. This makes

$$
\begin{aligned}
\operatorname{Hom}_{\mathbf{K}(R-\text { Mod })}(P, F) & =\operatorname{Hom}_{\mathbf{K}(R-\text { Mod })}\left(P, \mathscr{H o m}_{\mathbb{Z}}(I, \mathbb{Q} / \mathbb{Z})\right) \\
& =\operatorname{Hom}_{\mathbf{K}(\mathbb{Z})}\left(I \otimes_{R} P, \mathbb{Q} / \mathbb{Z}\right) \\
& =\operatorname{Hom}_{K(\mathbb{Z})}\left(\mathscr{H} o m_{R}\left(P^{*}, I\right), \mathbb{Q} / \mathbb{Z}\right) .
\end{aligned}
$$

Construction 5.4. - Now let $Q$ be a compact object in $\mathbf{K}(R-$ Proj). We remind the reader that, in [12, Proposition 7.12], we classified the compact objects in $\mathbf{K}(R-\operatorname{Proj})$. Replacing $Q$ by an isomorph if necessary, we may assume that $Q \in T$; that is, $Q$ is a bounded below chain complex of finitely generated, projective left $R$-modules. This means that $Q^{*}$ is a bounded above chain complex of finitely generated, projective right $R$-modules. Since $Q$ is compact, we furthermore know that $H^{i}\left(Q^{*}\right)=0$ if $i \ll 0$. We can choose a bounded below complex of injective right $R$-modules, quasi-isomorphic to $Q^{*}$; choose one, and denote it $I=I(Q)$. Let us choose and fix an $I(Q)$, for every compact object $Q$ in $\mathbf{K}(R-$ Proj). Clearly $I(Q)$ is a test-complex.

Proposition 5.5. - Let $R$ be a coherent ring. Let $\left\{I(Q), Q \in \mathbf{K}(R-\operatorname{Proj})^{c}\right\}$ be all the test-complexes of Construction 5.4. Then the objects $F(Q)=\mathscr{H}^{\circ} m_{\mathbb{Z}}(I(Q), \mathbb{Q} / \mathbb{Z})$ cogenerate the category $\mathbf{K}(R-$ Proj $) \cong \mathbf{K}(R-$ Flat $) / \mathscr{S}$.

Proof. - First of all, because $R$ is coherent, the objects $F(Q)$ belong to $\mathbf{K}(R$-Flat).

Let $P$ be any object in $T$, and assume $Q$ is a compact object. Both $P$ and $Q$ are chain complexes of finitely generated, projective $R$-modules; hence

$$
\mathscr{H}_{R}(Q, P)=\mathscr{H}_{R} m_{R}\left(P^{*}, Q^{*}\right) .
$$

Now $P^{*}$ is a bounded above chain complex of projectives, and hence the quasi-isomorphism $Q^{*} \longrightarrow I(Q)$, of Construction 5.4, induces a quasi-isomorphism

$$
\mathscr{H}_{\mathrm{om}}\left(P^{*}, Q^{*}\right) \longrightarrow \mathscr{H}_{\mathrm{om}}\left(P^{*}, I(Q)\right) \text {. }
$$

Since the abelian group $\mathbb{Q} / \mathbb{Z}$ is injective, we have an isomorphism of abelian groups

$$
\begin{gathered}
\operatorname{Hom}_{\mathbb{Z}}\left(\operatorname{Hom}_{\mathbf{K}(R-\text { Proj })}(Q, P), \mathbb{Q} / \mathbb{Z}\right) \\
\| \\
\operatorname{Hom}_{\mathbf{K}(\mathbb{Z})}\left(\mathscr{H o m}_{R}(Q, P), \mathbb{Q} / \mathbb{Z}\right) \longrightarrow \operatorname{Hom}_{\mathbf{K}(\mathbb{Z})}\left(\mathscr{H} o m_{R}\left(P^{*}, I(Q)\right), \mathbb{Q} / \mathbb{Z}\right) .
\end{gathered}
$$

Lemma 5.3 tells us that

$$
\operatorname{Hom}_{\mathbf{K}(\mathbb{Z})}\left(\mathscr{H} o m_{R}\left(P^{*}, I(Q)\right), \mathbb{Q} / \mathbb{Z}\right)=\operatorname{Hom}_{\mathbf{K}(R-\text { Flat })}(P, F(Q)) .
$$

Combining these isomorphisms, we have an isomorphism, natural in $P$,

$$
\operatorname{Hom}_{\mathbb{Z}}\left(\operatorname{Hom}_{\mathbf{K}(R-\text { Proj })}(Q, P), \mathbb{Q} / \mathbb{Z}\right) \longrightarrow \operatorname{Hom}_{\mathbf{K}(R-\text { Flat })}(P, F(Q)) .
$$


These are isomorphisms for all $P \in T$, in particular for all compact objects $P$. On the subcategory $\mathbf{K}(R-\text { Proj })^{c}$, of the compact objects in $\mathbf{K}(R-$ Proj $)$, the functor represented by $F(Q)$ is the functor taking $(-)$ to

$$
\operatorname{Hom}_{\mathbb{Z}}\left(\operatorname{Hom}_{\mathbf{K}(R-\text { Proj })}(Q,-), \mathbb{Q} / \mathbb{Z}\right) .
$$

This is an injective object in the abelian category $\mathscr{C} a t\left(\left\{\mathbf{K}(R-\operatorname{Proj})^{c}\right\}^{\text {op }}, \mathscr{A} b\right)$ of additive functors $\left\{\mathbf{K}(R-\operatorname{Proj})^{c}\right\}^{\text {op }} \longrightarrow \mathscr{A} b$. If we take the product, over all compact objects $Q \in \mathbf{K}(R-$ Proj $)$, we discover that the functor represented by

$$
F=\prod_{Q \in \mathbf{K}(R-\operatorname{Proj})^{c}} F(Q)
$$

is an injective cogenerator in $\mathscr{C} a t\left(\left\{\mathbf{K}(R-\mathrm{Proj})^{c}\right\}^{\text {op }}, \mathscr{A} b\right)$. By [12, Proposition 7.14] the category $\mathbf{K}(R-$ Proj $)$ is compactly generated. The argument above shows that $F$ is a BrownComenetz object in $\mathbf{K}(R-$ Proj). By [11, Theorem 8.6.1], $F$ cogenerates.

\section{REFERENCES}

[1] D. Bravo, E. Enochs, A. Iacob, O. Jenda, J. Rada, Cotorsion pairs in C( $R$-Mod $)$, to appear in Rocky Mountain J. of Math.

[2] E. H. J. Brown, Cohomology theories, Ann. of Math. 75 (1962), 467-484.

[3] E. E. Enochs, J. R. García Rozas, Flat covers of complexes, J. Algebra 210 (1998), 86-102.

[4] S. Iyengar, H. Krause, Acyclicity versus total acyclicity for complexes over Noetherian rings, Doc. Math. 11 (2006), 207-240.

[5] P. Jørgensen, The homotopy category of complexes of projective modules, Adv. Math. 193 (2005), 223-232.

[6] H. Krause, A Brown representability theorem via coherent functors, Topology 41 (2002), 853-861.

[7] H. Krause, The stable derived category of a Noetherian scheme, Compos. Math. 141 (2005), 1128-1162.

[8] H. Krause, Approximations and adjoints in homotopy categories, preprint arXiv:1005.0209.

[9] D. S. Murfet, The mock homotopy category of projectives and Grothendieck duality, Ph.D. Thesis, Australian National Univ., 2008.

[10] A. Neeman, Brown representability for the dual, Invent. Math. 133 (1998), 97-105.

[11] A. Neeman, Triangulated categories, Annals of Math. Studies 148, Princeton Univ. Press, 2001.

[12] A. Neeman, The homotopy category of flat modules, and Grothendieck duality, Invent. Math. 174 (2008), 255-308.

[13] A. Neeman, Brown representability follows from Rosický's theorem, J. Topol. 2 (2009), 262-276.

$4^{\mathrm{e}}$ SÉRIE - TOME $44-2011-\mathrm{N}^{\circ} 4$ 
[14] A. Neeman, Some adjoints in homotopy categories, Ann. of Math. 171 (2010), 2143 2155.

[15] J. RosickÝ, Generalized Brown representability in homotopy categories, Theory Appl. Categ. 14 (2005), 451-479.

[16] M. SAORÍN, J. ŠŤOvíčEK, On exact categories and applications to triangulated adjoints and model structures, preprint arXiv:1005.3248.

(Manuscrit reçu le 26 octobre 2009; accepté, après révision, le 29 novembre 2010.)

\footnotetext{
Amnon Neeman

Centre for Mathematics and its Applications

Mathematical Sciences Institute

John Dedman Building

The Australian National University

Canberra, ACT 0200, Australia

E-mail: Amnon.Neeman@anu.edu.au
} 